\title{
Economic Processes in the Conditions of Digitalization of Modern Society
}

\author{
Zhanna Bakaeva ${ }^{1, *}$, Alexey Stepanov ${ }^{1}$, Ella Shchegoleva $^{1}$, Sergey Imyarekov ${ }^{2}$, and Irina \\ Steklova $^{3}$ \\ ${ }^{1}$ Chuvash State University named after I.N. Ulyanov, 428015 Cheboksary, Russia \\ ${ }^{2}$ Saransk Cooperative Institute (branch) of the Russian University of Cooperation, Saransk, Russia \\ ${ }^{3}$ Saratov State Technical University named after Yu.A. Gagarin, 410054 Saratov, Russia
}

\begin{abstract}
The article deals with the problem of digitalization in the economic processes of modern societies. The fundamental aspects of economic processes are the development international trade and international services. Communications define "proprietary" technologies as factors of Informatization and digitalization of society. An important element of building "smart" computing systems is artificial intelligence. Creating the concept of artificial intelligence has its own shortcomings, which are reflected in the allocation ethical norms and principles of the financial environment modern society. The effectiveness of the digital environment is determined by the principle of "successful functioning" of the subject, taking into account the temporary accumulation goods and services in globalization and digitalization. Research activities contribute to the development digitalization of economic processes in the structure digitalization society.
\end{abstract}

\section{Introduction}

According to modern research, the core of the digital economy is considered to be the creation big data products and the offer of services associated with numerical technologies. State statistics, regardless of its changes, determine an increase in the volume of trade in products in the structure of the digital economy (in the usual approximation, about $4 \%$ ), the size of services provided in this area increases (up to 30\%). The main components of the digital economy are considered to be inter-net trade and payments, advertisements, and the entertainment network. The increase in the company's expenses caused by such technologies indicates the importance digitalization in the structure innovations. An easily accessible numerical infrastructure is being formed that improves the interconnection technologies (for example, fiber-visual concepts of data transmission). They reduce the cost of maintaining mobile connectivity and expand the use of special devices in order to allow them to enter the Internet network. As a result of the technology [1].

\footnotetext{
*Corresponding author: jannasar@yandex.ru
} 


\section{Materials and methods}

Economic processes are considered in the integration all elements that make up a set digital structures. Consistency and classification are among the defining methods economic systems. This is due to the specific use of such methods in various areas interdisciplinary research. Modeling and forecasting analyze the properties various economic systems [2].

Highly specialized methods are considered as defining and describing economic processes within a certain time frame. An integrated approach is a single method that combines various types processes with certain economic characteristics. The main methods digitalization determine the relationship numerical values.

Logical, comparative and analytical approaches determine the digitalization of the economy, which depends on the management organization. Great opportunities for digital representation information lead to the fact that digitalization forms integral technological environments (ecosystems, platforms), within which the user can create the necessary environment for himself (technological, instrumental, methodological, documentary, partner, etc. [3].

\section{Results and discussion}

Digitalization is seen as the next wave of machine learning using digital tools. Starting from the theoretical and practical foundations of modern computing in the 30-40s, computers have become an indispensable tool for many scientific processes of cognition. They made "big science" possible and allowed the creation new fields that relied heavily on computer modeling. At the broad social level, the use of information technologies is not something new that arises as a result of the automation of production processes. However, the key differences between today's digital transformation and previous use computer technology are the result a combination of advances in machine learning, increased data availability, and computing power.

"End-to-end" technological processes that radically change the situation in the market segments modern economic development. The technologies of the numerical economy include: big data, neurotechnologies, artificial intelligence, concepts the numerical register (blockchain), the latest production processes, and the production network Internet, elements of robotics, sensors, technological processes, wireless interconnection, technological processes conditional reality. Two trends in the formation artificial intelligence are emphasized. The first is related to the resolution issues related to the approximation artificial intelligence concepts, as well as their integration. in the concept of General, synthetic intelligence. Areas use artificial intelligence are determined by automated data transfer, the concept informative security, and the acquisition specialists. Definition; robotics, expert concepts; the study of mappings in the structure of the numerical economy determines the main digital trend in the development of society [3].

Artificial intelligence systems are increasingly solving problems where traditional computer programs fail. Artificial intelligence is a concept of quantitative Sciences that deals with automating intelligent behavior using digital concepts from other cognitive Sciences. Since the concept of artificial intelligence itself is relatively diverse and there is no clear scientific definition of it. Technical innovations have changed the assessment of artificial intelligence to a certain extent. Machine learning has become the basic technology for self-driving cars, robots that help automate social and economic processes. Technological progress, training of qualified practitioners, and competitive pressures are accelerating the spread of artificial intelligence. Accordingly, in recent years, discourses have developed that serve as guidelines for assessing the potential impact of artificial intelligence and big data in scientific practice: the black box Problem: in contrast to 
"classical" computer algorithms, is determined by deep neural networks using various programming methods. Instead of clear program structures, a neural network is used, its connectivity over a huge number of training cycles. Eventually, the algorithm comes up with a solution to the obvious physical or logical basis of the object. Therefore, some algorithms look like "black boxes", which is a significant limitation of their practical application, provided that the subject automates machine translation of any digital system.

Unlike explicitly written programs, new types of artificial intelligence systems (socalled deep learning algorithms) are trained on a variety of examples and form the procedure for making the right decisions. Computer-based learning mimics human learning to some extent, although the latter involves emotional engagement and commitment. Over the course of several years, these advances have allowed artificial intelligence systems to achieve impressive success in complex and ambiguous tasks such as image recognition, translation, radiological image analysis, and games. They not only compete with human abilities, but sometimes even surpass them. These methods are rapidly being improved and applied by subjects who are involved in the diagnosis of a particular type of disease.

Thus, artificial intelligence becomes a favorable technology for a wide range of applications. Until recently, subjects defined algorithmically controlled tasks, created the necessary programs, and applied these algorithms in certain areas. But now that large amounts of diverse data form the basis of a problem context, machine learning generates a problem-solving algorithm that is then used in heterogeneous scientific applications.

Thus, "feedback" is carried out between the solution of a specific problem using an algorithm in a specific application area and its adaptation in the context of restriction and control. The result of this application is a gradual transition from decision support using automation algorithms to areas related to the quality of life [4].

The next important point in the process of digitalization in economic processes is to establish the limits of ethical access to information. This is a difficult question for various stakeholders, companies, and social institutions in the structure of digital economic systems. The main problem that determines the information generated by numerical technologies is considered to be depersonalization of providing medical information. The main moral problem of digitalization is determined by the choice of public companies, communities, the latest products and services that are directly associated with the use of individual information. The greatest number of risks appear when collecting information and are concentrated in three areas:processing information with support for artificial intelligence, surveillance, and monitoring on the Internet. A special manifestation of digital ethics is financial ethics ("business ethics", "business ethics"). Much attention is currently being paid to this issue. Well-being in financial ethics is considered as a material reward for conscientious activity. The essence of financial ethics lies in the idea that the created result is not implemented in any way in "financial theory". The ethical and financial concept is conditioned by the possibility to form a society of objects and resources [5].

Digital data is a Central resource for artificial intelligence algorithms. However, depending on the type of problem being solved, one-sidedness is formed in digital data, which determines the behavior of software algorithms. The emergence of digital science in modern Europe was accompanied by the development of values that characterize the "ethical processes" in digital research [6]. They are referred to as "digital epistemological norms" and practice knowledge of economic processes through normative documents that are internalized by appealing to values and pragmatic effectiveness in providing digital knowledge. Digital norms are not time-stable and cannot be clearly separated from other types of documents. Of course, they are crucial for practical digital science. To gain solid scientific digital knowledge, researchers must be able to think about and analyze the digital objects under study [7]. 
The ontology of digital norms related to scientific research, including collegiality, foresight, imagination, visualization or conceptualization of abstract entities (openness, receptivity) is crucial in modern society. Of course, all these criteria for digitalization are important requirements for the subject's activity, when digital assessment is ahead of research [8]. These digital standards are directly related to the discovery of empirical ideas about the digitalization of reality [9]. Skepticism and objectivity are important scientific criteria for digital governance.

Since the goal of digitalization is to evaluate changes in scientific practice based on digital criteria of technological developments. There is a problem with the practice of digital cognition, which is widely discussed in the reasons for scientific changes in digitalization. The answer to this problem requires addressing both macro-scale economic factors and micro-factors, i.e., specific research in experimental economic systems. The ongoing digital transformation of the economy affects both the macro and micro level of the subject's activity in the field of digitalization. The potential positive and negative use of new digital tools in scientific practice in relation to the criteria of digitalization and Informatization of knowledge is outlined [10].

When forming a digital economy, target or "problem" characteristics are identified, which contain several points. First, it is the number of specialists trained in the field of information and telecommunications technologies [11]. This representation of today's economic activity contributes to the formation of the concept of digital infrastructure. Second, the stated principle of "successful digital activity" also contributes to the creation of ecosystem-based and competitive digital operators in global markets. Although this point is quite difficult to implement in the circumstances of the current reality, since the main guideline for the formation of the country's economy is the export of oil and gas materials. Third, the principle of "successful operation of computing technologies and provision of digital services" will be a problematic point of digitalization [12].

In the Russian Federation, the concept of R \& d is a complex infrastructure, as well as the principle of scientific and technological progress in state corporations and municipal structures within the framework of Informatization. Despite the disadvantages, there are positive.

\section{Conclusions}

A problematic activity in the digital economy is the principle of "successful functioning", which is associated with small companies in the field of numerical technologies, as well as a platform for providing digital services. In the Russian Federation, the concept of $\mathrm{R} \& \mathrm{~d}$ is important, as well as the analysis of expenditures in this area. The principle of accumulation of digital services in state corporations and municipal structures is used to implement more complete and effective management of digital systems.

Despite the shortcomings listed above, there are several positive aspects associated with the transformation of digitalization and improving costs and reducing real transactions in the direction of increasing conditional ones, which significantly reduce the cost of mobile communications. According to the plan for the development of digital economic processes, funds are released, which are determined by the adopted regulatory legal acts. They provide elastic regulation of relationships in the structure of large data sets. The specific dangers of digitalization are reflected in the concepts of big data generation.

In turn, in order to implement digitalization projects, we can note the guarantees of social and financial requirements, which at the current stage are accelerating and changing in such a way that they transform production relationships and the composition of economic structures. Emerging economic information in the digital process system changes regularly and requires a variety of applications. 
In order to effectively operate the economy of large numbers, it is necessary to change the concept of urban governance with the transition to a digitization platform for providing services, as well as training competent employees and creating a reference and innovative infrastructure, including technology parks, as well as "incubators" of entrepreneurship. Digitalization also involves the formation of a digital company (through the units of functioning of Commerce), which implies the implementation of production and administrative actions in the numerical format of the present period.

Taking into account the above, we can talk about natural processes that reflect the risks of monitoring the activities of participants in digitalization. Consequently, the significance of state sovereignty in the cross-border state of the economy of large numbers is being reviewed. The threat to the informational security of the state and its structures can be traced to a reduction in the degree of employment, lack of work, reduced skills of subjects, as well as the intellectualization of society.

The expected changes in the field of digitalization are reflected in the concepts of creating theoretical foundations for these processes. A special place is given to city management and financial changes in the industry. The problem of limiting in the context of digitalization becomes important in the short period of its implementation. The main results are considered to be economic processes that are being transformed in the structure of digitalization of the modern economy.

\section{References}

1. M. F. Ananiev, International J. of Recent Technology and Engineering, 2 (2019)

2. B. Akhmadeev, S. Manakhov, J. of security and sustainable development, 5 (2015)

3. Z. Y. Bakaeva, Ekoloji, 107 (2019)

4. S. Bodrunov, Proceedings of the European Conference on Intellectual Capital, 1 (2019)

5. R. Bucht, R. Hicks, Digital leadership Strategies, 10 (2019)

6. G. Kalinina, Modern j. of language teaching methods, 5 (2017)

7. G. V. Kalinina, Prawo i Więź, 3 (2019)

8. I. A. Zaraychenko, A. I. Shinkevich, M. Y. Mitrofanova, T. I. Ladykova, Y. V. Nuretdinova, G. M. Kharisova, M. A. Zhukova, G. M. Kharisova, J. of Advanced Research in Law and Economics, 1 (2017)

9. M. N. Lukyanova, World j. of applied Sciences, 30 (2014)

10. M. A. Gorbunov, A. V. Fadeeva, V. B. Shirshikov, P. A. Matveev, J. V. Popova, M. Y. Mitrofanova, Z. Y. Bakaeva, N. A. Mashkin, Ekoloji, 107 (2019)

11. N. A. Moiseev, J. of statistical computing and modeling, 87 (2017)

12. N. P. Savina, Russian economic online magazine, 4 (2013) 